\title{
Epidemiological associations between iron and cardiovascular disease and diabetes
}

\author{
Debargha Basuli ${ }^{1}$, Richard G. Stevens ${ }^{2}$, Frank M. Torti ${ }^{3}$ and Suzy V. Torti ${ }^{1}$ * \\ ' Molecular Biology and Biophysicis, University of Connecticut Health Center, Farmington, CT, USA \\ ${ }^{2}$ Division of Epidemiology and Biostatistics, Department of Community Medicine and Health Care, University of Connecticut Health Center, Farmington, \\ CT, USA \\ ${ }^{3}$ Internal Medicine, University of Connecticut Health Center, Farmington, CT, USA
}

\section{Edited by:}

Paolo Arosio, University of Brescia, Italy

\section{Reviewed by:}

Luca Valenti, Università degli Studi di Milano, Italy

Igor Theurl, Medical University of Innsbruck, Austria

\section{*Correspondence:}

Suzy V. Torti, Molecular Biology and Biophysicis, University of Connecticut Health Center, 263 Farmington

Avenue, Farmington, CT 06030, USA e-mail:storti@uchc.edu
Disruptions in iron homeostasis are linked to a broad spectrum of chronic conditions including cardiovascular, malignant, metabolic, and neurodegenerative disease. Evidence supporting this contention derives from a variety of analytical approaches, ranging from molecular to population-based studies. This review focuses on key epidemiological studies that assess the relationship between body iron status and chronic diseases, with particular emphasis on atherosclerosis , metabolic syndrome and diabetes. Multiple surrogates have been used to measure body iron status, including serum ferritin, transferrin saturation, serum iron, and dietary iron intake. The lack of a uniform and standardized means of assessing body iron status has limited the precision of epidemiological associations. Intervention studies using depletion of iron to alter risk have been conducted. Genetic and molecular techniques have helped to explicate the biochemistry of iron metabolism at the molecular level. Plausible explanations for how iron contributes to the pathogenesis of these chronic diseases are beginning to be elucidated. Most evidence supports the hypothesis that excess iron contributes to chronic disease by fostering excess production of free radicals. Overall, epidemiological studies, reinforced by basic science experiments, provide a strong line of evidence supporting the association between iron and elevated risk of cardiovascular disease and diabetes. In this narrative review we attempt to condense the information from existing literature on this topic.

Keywords: iron, cardiovascular disease, diabetes mellitus, metabolic syndrome, epidemiologic studies

\section{INTRODUCTION}

Cardiovascular disease (CVD) and diabetes are major health problems worldwide. In the United States, approximately one in four deaths are due to heart disease, making it the leading cause of death for both men and women (Heron et al., 2009; Heidenreich et al., 2011) ${ }^{1}$. Coronary heart disease is the most common type of heart disease, and costs the US over 100 billion each year (Go et al., 2013). Risk factors include high blood pressure, high LDL cholesterol, smoking, diabetes, and obesity (MMWR Morb Mortal Wkly Rep 60, 2001). Diabetes is itself a significant health problem that is reaching epidemic proportions, with a global prevalence of 382 million people in 2013. It is estimated that by 2035 this will rise to 592 million $^{2}$.

The search for risk factors and methods of prevention for both CVD and diabetes are major efforts of the medical and research community. The role of iron as a risk factor for CVD and diabetes has drawn attention in part due to the concept that it may be a risk factor susceptible to simple dietary modification. Although this is an oversimplification, many (not all) reports suggest that there is indeed an association between iron and both CVD and diabetes, as detailed in this review.

\footnotetext{
${ }^{1}$ http://www.cdc.gov/heartdisease/facts.htm (accessed February 2, 2014).

${ }^{2} \mathrm{http}: / / \mathrm{www}$. idf.org/diabetesatlas
}

Epidemiological studies have been a powerful tool to probe the association between iron and CVD and diabetes. Several types of study design have been employed. Each of these has its benefits and limitations. Briefly, epidemiological studies can be divided into observational and experimental studies. The difference between an observation and experimental study is that in the latter, an outcome is studied in a population in the absence or presence of an intervention by the investigator. In an observational study, there is no intervention and the investigator simply "observes" and analyses the relationship between exposure and disease outcome. Observational studies include cohort studies, case-control studies and cross sectional studies. A cohort study is an analysis of risk factors where a disease-free study population is identified and followed prospectively over time and a subsequent evaluation is done to find the association between the exposure and disease outcome. While this kind of study can provide strong scientific evidence of an association between risk factors and disease and a temporal framework to assess causality, it is limited by the requirement for a large sample size and long follow-up duration. Often several biases can adulterate the evidence. A case control study on the other hand starts with groups with and without an outcome and evaluates how much a suspected exposure might have contributed to the present outcome status. Thus in comparison to cohort studies, case control studies are 
relatively quicker to conduct, inexpensive and require comparatively fewer subjects. Cross sectional studies collect and analyze the data on exposure and disease at one specific time point. Such studies cannot evaluate cause and effect relationships since there is no temporal assessment. Table 1 shows the level of evidence of different types of epidemiological studies. In the hierarchy of evidence-based medicine, experimental studies (or more specifically randomized controlled trials) are recognized as level I of scientific evidence. However, the consensus over this has recently changed as observational studies have been reported to be as effective as randomized controlled trials in estimating the impact of medical interventions on disease outcomes (Benson and Hartz, 2000; Concato et al., 2000). Of course, it must also be emphasized that for a potential hazard, such as elevated body iron, a randomized controlled trials cannot be performed ethically, although it could be done for a study of iron reduction by, for example, phlebotomy.

Virtually all of these epidemiological analyses have been used to probe the relationship between iron and CVD or diabetes. PubMed searches using the terms "iron heart disease epidemiology" or "iron diabetes epidemiology" identify over 500 papers for each search term. In this narrative review, we have not attempted to be comprehensive, but to focus on key epidemiological studies that have investigated these issues. We provide some historical context, but emphasize recent, well-controlled studies with large sample size.

\section{IRON AND CARDIOVASCULAR DISEASE}

Cardiovascular disease is a broad term that includes ischemic and non-ischemic irregularities. Association with iron has been mainly studied and found in ischemic cardiovascular diseases caused by atherosclerosis. To measure ischemic disease outcome, several different endpoints have been used, including coronary heart disease (CHD), carotid artery plaque formation, coronary artery calcium deposition, carotid intima thickness, and atherosclerosis. CHD has been measured by myocardial infarction and cardiogenic angina occurrences and deaths from such incidents. The role of iron in CVD has generally been explored in a group of individuals using one of these defined endpoints. For the purposes of this review, we have included all of these clinical entities under the umbrella of CVD and have not attempted to differentiate among them.

The most common measurement used in the assessment of body iron has been serum ferritin. Serum ferritin was shown to correlate with body iron stores in the 1970s, and is still used clinically for this purpose (Jacobs et al., 1972; Cook et al., 1974; Jacobs and Worwood, 1975; Wang et al., 2010). However, serum ferritin can also be elevated by acute and chronic inflammation (Wang et al., 2010). Accounting for the contribution of these variables thus becomes an important component of studies that use serum ferritin as a measure of body iron, as discussed below. Less frequently, the ratio of soluble transferrin receptor to ferritin has been used, as it has been suggested that this is a more precise measure for body iron store than ferritin alone (Skikne et al., 1990). Catalytically available iron has also been measured in some studies, with the goal of measuring reactive rather than total iron. This approach derives from the consideration that the preponderance of iron in the body is bound to proteins and is not available for participation in the potentially deleterious reactions that are thought to underlie much of the toxicity of iron, such as the formation of reactive oxygen species. A limitation of this approach is that since catalytically available iron represents a relatively small fraction of total iron, its measurement is technically challenging.

The hypothesis that iron status could influence the risk of coronary heart disease was first proposed by Sullivan in the 1980s. Sullivan hypothesized that the higher occurrence of CHD in men and post-menopausal women than in pre-menopausal women was due to higher iron stores in them compared to menstruating women (Sullivan, 1981, 1989). Some earlier studies supported the hypothesis. In a cohort of 2873 Framingham women, an increase in incidence of CHD and disease severity was observed in women who had either natural or surgical menopause (Gordon et al., 1978). In some early prospective studies, a weak association between high blood hemoglobin and hematocrit and risk of CHD was noted (Cullen etal., 1981; Bottiger and Carlson, 1982; Knottnerus et al., 1988). Hemoglobin and hematocrit are not good surrogates for body iron status and during this period serum ferritin was emerging as the best measurement of body iron status (Cook et al., 1974; Kaltwasser and Werner, 1989). The first report in humans on the association between serum ferritin and CHD risk was published in Salonen et al. (1992a). In this cohort of randomly selected 1931 Eastern Finnish men, serum ferritin concentration had a significant association with ischemic heart disease risk. Subjects with serum ferritin $\geq 200 \mu \mathrm{g} / \mathrm{l}$ had a 2.2-fold (95\% CI, 1.2-4.0; $p<0.01$ ) higher risk of acute myocardial infarction compared to men with lower serum ferritin. Total blood leucocyte count was adjusted in the statistical analysis to rule out the potential confounding effect of inflammation or chronic

Table 1 | Evidence level provided by epidemiological studies of different design.

\begin{tabular}{ll}
\hline Level of evidence & Qualifying studies \\
\hline I & High quality, multicenter or single center, randomized controlled trial with adequate power: or systemic review of these studies \\
II & Lesser quality, randomized controlled trial; prospective cohort study; or systemic review of these studies \\
III & Retrospective comparative study; case-control study; or systemic review of these studies \\
IV & Case series \\
V & Expert opinion; case report or clinical example; or evidence based on physiology, bench research, or "first principles"
\end{tabular}

Song and Chung (2010). 
vascular disease that would elevate serum ferritin independent of body iron status. The association was stronger in men with higher concentrations of low density lipoproteins $(\mathrm{RR}=1.8,95 \% \mathrm{CI}$, $0.9-3.5$, NS in men with low LDL and a RR $=4.7,95 \% \mathrm{CI}$, 1.4-16.3, $p<0.05$ in men with high LDL). After this report, the group conducted another nested case-control study within the Kuopio Ischemic Heart Disease Risk Factor Study (KIHD) cohort and found that men with high body iron stores were at increased risk of acute myocardial infarction (AMI), confirming their original observation (Tuomainen et al., 1998). In this study body iron status was measured by ratio of soluble TfR and ferritin which some authors suggest as a better measure of body iron than serum ferritin alone (Cook etal., 1974; Skikne etal., 1990).

The first prospective study in women was conducted in 11,471 Dutch post-menopausal female subjects aged 49-70 years (van der et al., 2005). In the study, the multivariate hazard ratio of ischemic strokes in the highest tertile of serum ferritin concentration was 2.23(95\% CI, 1.05-4.73) compared to the lowest. An interesting finding common to some of these studies was the interaction of LDL with serum ferritin in increasing the risk of ischemic events. A plausible biological mechanism underlying this interaction may be the ability of iron to produce reactive oxygen species. Iron catalyzes the Fenton reaction which produces potent oxidants that increase the risk of atherosclerosis by promoting the peroxidation of lipids (McCord, 1991; Salonen et al., 1992b; Berliner and Heinecke, 1996). Local release of iron from ferritin by superoxide radical generated by ischemia/reperfusion injury to blood vessels may further exacerbate this damage (Thomas et al., 1985).

Many of the studies discussed above focused on cardiovascular events such as acute myocardial infarction. However, myocardial infarction is a complex endpoint resulting from multiple potential pathogenesis pathways. To circumvent this limitation, other studies used preclinical atherosclerosis as the dependent variable and explored its relationship to serum ferritin. For example, Kiechl et al. (1997) reported that serum ferritin level was closely related to incidence of carotid atherosclerosis and progression of previous atherosclerotic lesions in a cohort of Italian men and women. In a cross sectional study that included German men and women, there was an association of serum ferritin with carotid plaque prevalence in both men (OR: 1.33; 95\% CI, 1.08-1.44) and women (OR, 1.29; 95\% CI, 0.98-1.75) (Wolff et al., 2004). When the study population was divided into ferritin octiles, both men and women showed a dose-dependent relationship between serum ferritin and atherosclerotic plaques. Subjects with malignancy and liver diseases were excluded to eliminate the confounding effect of inflammation and mild liver disease, but no adjustments were made for any inflammatory markers. Thus, acute or chronic inflammatory conditions could have confounded the findings in this study by affecting serum ferritin levels at the time of measurement.

Several recent studies have shown that serum ferritin is independently associated with preclinical measures of vascular diseases. Sung et al. (2012) showed that ferritin levels in a large cohort of 12,033 young Korean men were independently associated with coronary artery calcium content, a marker of early coronary artery sclerosis. In a similar study, Valenti et al. (2011) showed that in a small study population of non-alcoholic fatty liver patients, carotid intima medial thickness and carotid plaque were independently associated with increased ferritin levels.

The potentially damaging effect of iron on the heart, pancreas, liver and other organs was made evident in part through the study of hemochromatosis, a disorder in which excess iron is absorbed and deposited in tissues. Patients with untreated hemochromatosis can exhibit diabetes, liver damage, and cardiac injury among other symptoms (Wolff, 1993; Witte et al., 1996; Powell et al., 1998). Mutations in HFE gene (the hemochromatosis gene) are one cause of hemochromatosis. Using a mouse model for hereditary hemochromatosis, Turoczi et al. (2003) showed an interaction of dietary iron intake and HFE gene status (KO vs. wildtype) in degree of ischemia/reperfusion injury to heart; HFE KO mice showed greater ventricular dysfunction, myocardial infarct size, and cardiomyocyte apoptosis compared to wild type mice on a standard diet, and an even greater degree of damage in the HFE KO mice fed a high iron diet (Turoczi et al., 2003). In human subjects, a similar increase in cardiovascular death was observed in women heterozygous for the HFE gene (Roest et al., 1999). However, no association between the HFE genotype and atherosclerosis has been found in hemochromatosis patients in spite of iron overload status in these patients (van der et al., 2006; Engberink et al., 2010). Valenti et al. (2011) found that the prevalence of carotid plaques was highest in patients with hyperferritinemia independent of HFE genotype. Although the risk of atherosclerotic heart disease appears unrelated to HFE genotype, hemochromatosis patients do have a higher risk of iron-related non-ischemic cardiovascular irregularities (Gaenzer et al., 2002; Dunn et al., 2008).

Multiple mechanisms likely underlie the association of iron with CVD. In addition to the ability of iron to promote lipid peroxidation, recent studies have implicated the peptide hormone hepcidin in atherosclerosis. Hepcidin is a central regulator of iron absorption and recycling (Ganz, 2013). Hepcidin acts by binding ferroportin, an iron efflux pump present in both enterocytes and macrophages. Binding of hepcidin to ferroportin triggers ferroportin degradation (Nemeth etal., 2004), thus inhibiting the delivery of iron to the circulation through the enterocyte as well as inhibiting iron recycling in macrophages. Valenti et al. (2011) observed that serum hepcidin was independently associated with carotid plaques, suggesting that hepcidin-induced iron accumulation may be involved in the process of atherogenesis in subjects negative for HFE mutations. Specifically, hepcidin may induce excessive iron trapping within macrophages, resulting in an increase in oxidative stress, transformation into foam cells, and ultimately to atherosclerotic vascular disease (Sullivan, 2007, 2009; Theurl et al., 2008). This hypothesis is known as the "iron hypothesis" and was proposed by Sullivan (2009). Since hepcidin is decreased in patients with hereditary hemochromatosis, this model provides a potential explanation for the previous observation that atherosclerosis risk is not increased in subjects with hereditary hemochromatosis (van der et al., 2006). However, a recent experimental study provided evidence that hepatic hepcidin expression is not correlated with atherosclerosis progression in a mouse model Kautz etal. (2013). Further, the authors reported that increasing macrophage iron accumulation in mice 
with atherosclerosis either through a genetic mutation in the ferroportin gene or through parenteral iron administration failed to increase the size of atherosclerotic lesions or lesion calcification. The study challenged the "iron hypothesis."

An alternative approach to the use of serum ferritin to assess the relationship between iron and CVD has been to assess the relationship between catalytic iron and heart disease. Catalytic iron is the iron that is not bound to transferrin or ferritin and is available to take part in chemical reactions to produce oxidant products. This can be measured using a bleomycin detectable iron assay (BDI; von Bonsdorff et al., 2002). Results from such studies are equivocal. While some studies found a relation between catalytic iron with CVDs (Rajapurkar et al., 2012), a recent study with a larger study population failed to report any association of catalytic iron with risk of MI and recurrent ischemic events (Steen et al., 2013). However the study showed that in a cohort of 1701 patients with AMI or unstable angina, catalytic iron was associated with stepwise increase in all-cause mortality (multivariate adjusted $\mathrm{HR}=3.97$, 95\% CI 1.09-14.1, $p=0.036$, highest quartile vs. baseline) when followed for a median of 10 months. Although no significant association of ischemic events with iron was reported, most of the deaths were related to ischemic complications, and thus the contribution of catalytic iron could not be ruled out completely. It is to be noted that serum catalytic iron does not reflect the intraplaque iron which might be a more proximal factor for vascular ischemic injuries (Nelson et al., 1992; Castellanos et al., 2002). Unfortunately, these studies did not report the relationship between serum ferritin and outcome. It thus remains unaddressed whether or not catalytic iron is more strongly associated than serum ferritin with outcome.

Another approach to testing a potential link between iron and CVDs has been the study of dietary iron intake and risk. Zhang et al. (2012) reported that dietary intake of total iron was positively associated with deaths from strokes and CVD in a cohort of 23,083 Asian men with a multivariate hazard ratio of 1.43 (95\% CI, $1.02-2, p=0.009$ ) for stroke and 1.27 (95\% CI, 1.01-1.58, $p=0.023$ ) for CVD after adjustment for other CVD risk factors; iron intake was not associated with these outcomes in women, however. In another large prospective study in almost 50,000 European men, there was a positive association of dietary iron, more specifically heme iron (a form of iron that is more readily absorbed by the gut than inorganic iron), with strokes in a follow-up period of 11.7 years (Kaluza et al., 2013). Adjustments were made for red meat consumption to rule out confounding by other known risk factors for stroke such as $N$-nitroso compounds and heterocyclic compounds (Forstermann, 2008). The association was observed in normal weight men and not in overweight or obese men, most likely because of decreased iron absorption due to increased hepcidin in the chronic inflammatory state associated with obesity (Greenberg and Obin, 2006). Adipocyte hepcidin expression is known to be positively correlated with obesity (Bekri et al., 2006). This association is still to be properly evaluated in women.

Although the foregoing studies appear well-conducted, there are also a number of other well-conducted studies that have found no association of markers of body iron and risk of CVD (Baer et al., 1994; Danesh and Appleby, 1999; Gupta et al., 2000;
Knuiman et al., 2003; Sun et al., 2008b; Friedrich et al., 2009). Discordant results among studies may in part be due to imprecision in the surrogate markers used to measure iron status (ferritin, total iron binding capacity, transferrin saturation, serum iron, and dietary iron intake), which are all indirect measures of body iron stores. Because these variables are subject to temporal and measurement variations, there is undoubtedly exposure misclassification in the subjects. This non-differential misclassification would reduce the ability of a study to identify a true association should one exist. An additional potential problem, particularly in cross-sectional studies, is that observed elevations in serum ferritin may represent an effect rather than a cause of underlying pathology.

If iron is associated with CVD, can interventions that reduce iron reduce risk? Unfortunately, only a limited number of intervention studies have been conducted, but results of these studies are at least suggestive that modulating iron can reduce risk. In animal models, treatment with deferoxamine, an iron chelator, during ischemia improved recovery and reduced reperfusion-induced oxygen radical formation in rabbit hearts (Williams et al., 1991). Paraskevaidis etal. (2005) reported that deferoxamine infusion ameliorated lipid peroxidation and improved long term outcome in patients having coronary artery bypass surgery. In a recent randomized controlled single blinded study, Zacharski et al. (2011) showed that a lower ferritin level predicted improved outcome and iron reduction by phlebotomy improved outcomes by preventing or delaying non-fatal myocardial infarction and stroke in young age patients with peripheral artery disease.

\section{IRON AND METABOLIC SYNDROME AND DIABETES}

Metabolic syndrome refers to a collection of risk factors that increase the likelihood of heart disease, diabetes and stroke. They include a large waistline, high triglyceride, low HDL cholesterol, high blood pressure and high fasting blood sugar. The presence of three of these five risk factors, many of which are associated with obesity, is defined as metabolic syndrome ${ }^{3}$.

Multiple studies have shown that excess body iron is associated with one or more components of metabolic syndrome (Jehn et al., 2004; Bozzini et al., 2005; Choi et al., 2005; Gonzalez et al., 2006; Sun et al., 2008a). To study the association of iron with metabolic syndrome in normal individuals, a cross-sectional study in 6044 US adults was conducted. The results showed a significant association of ferritin level with metabolic syndrome and insulin resistance (IR) after excluding hemochromatosis cases and adjusting for age, race/ethnicity, C-reactive protein, smoking, alcohol intake, and BMI (Jehn et al., 2004). Other studies in western populations showed similar associations (Jehn et al., 2004; Bozzini et al., 2005; Gonzalez et al., 2006; Wrede et al., 2006). A positive association of serum ferritin with the prevalence of metabolic syndrome in a study population of 8441 people including both sexes and from different provinces of China was recently reported (Li et al., 2013). Mendler etal. (1999) showed that in a cohort of patients with unexplained hepatic iron overload, IR was also often observed. Such patients with non-alcoholic fatty liver disease also tend to have elevated ferritin levels (Valenti et al., 2003,

${ }^{3}$ http://www.nhlbi.nih.gov/health/health-topics/topics/ms/ 
2006, 2007, 2010) which is now considered a feature of metabolic syndrome (Marchesini et al., 2001, 2003; Angulo, 2002). The constellation of hepatic steatosis, mild to moderate iron overload in both hepatocytes and macrophages, increased serum ferritin levels, and insulin resistance is commonly referred to as dysmetabolic iron overload syndrome or DIOS (Barisani et al., 2008; Riva et al., 2008; Datz et al., 2013). DIOS is detected in about one third of the patients with NAFLD and MetS and may be predisposing factor to the development of type 2 DM and CVDs [Dongiovanni et al., 2011; Valenti et al., 2012; See Dongiovanni et al. (2011) and Datz et al. (2013) for detailed discussion and review of this topic]. Iron depletion in such patients has been shown to improve histological liver damage and abnormal liver function when compared to lifestyle modification (Valenti et al., 2014).

Similarly, many epidemiological studies have reported statistically significant associations of body iron with diabetes, although results from all studies are not entirely consistent. In one longitudinal study of overweight/obese individuals with an impaired glucose tolerance test, there was no association between ferritin levels with risk of diabetes (Rajpathak et al., 2009). In another similar study, adjustment for BMI and components of the metabolic syndrome produced a null result (Jehn et al., 2007). However, in a prospective study in China, a country with the largest diabetic population in the world, an almost twofold increased risk of type 2 diabetes was observed among middle aged and elderly persons in the highest quintile of ferritin level compared with those in lowest after adjusting for known risk factors including high sensitivity $\mathrm{C}$ reactive protein (hsCRP), BMI, $\gamma$-glutamyl transferase (GGT), and adiponectin (Sun et al., 2013). The result was consistent with findings from similar prospective cohort studies in Caucasian populations (Salonen etal., 1998; Jiang etal., 2004; Forouhi et al., 2007; Montonen et al., 2012). In the EPIC (European Prospective Investigation of Cancer)-Norfolk cohort, serum ferritin was an important and independent predicting factor for development of diabetes after adjustment for conventional risk factors as well as vitamin C levels, CRP, IL6, liver function test (ALT, GGT), fibrinogen and adiponectin (Forouhi et al., 2007). In a case cohort study among 27,548 participants of the EPIC Postdam study in Germany, the sTFR-ferritin ratio was significantly inversely related to the risk of type 2 diabetes, and ferritin concentration was associated with higher risk (Montonen et al., 2012). The result was independent of biomarkers of inflammation, hepatic fat, IR, and dyslipidemia.

In evaluating these studies, a few considerations must be borne in mind. In epidemiological studies investigating the relation between iron and diabetes, serum ferritin is the most commonly used indicator of body iron stores. As mentioned in the introduction, the use of ferritin in assessing body iron stores has been somewhat challenging because ferritin can be elevated in inflammation, cancer, and liver disease (Wang et al., 2010). Serum ferritin concentration can also be increased in some conditions like obesity and metabolic syndrome which are associated risk factors for type 2 diabetes (Lee et al., 2009). It thus becomes difficult to discern whether the association of ferritin with diabetes is due to other concomitant conditions or serum ferritin levels increase as a result of diabetes (a case of reverse causation). In addition, serum ferritin has been correlated with dyslipidemia biomarkers (Halle et al., 1997), hepatic enzymes (Choi et al., 2005), and negatively associated with adiponectin, an insulin sensitizing adipokine that is decreased in diabetic patients (Forouhi et al., 2007). Thus adjustments for these components become very important.

The exact molecular mechanism of iron-related pathology in metabolic syndrome and diabetes is not clearly understood. Iron is a powerful pro-oxidant and can cause cellular damage by producing reactive oxygen species in different tissues of the body (Andrews, 1999; Rajpathak et al., 2009). Insulin producing pancreatic $\beta$ cells have been shown to be particularly susceptible to oxidative injury, in part due to decreased expression of antioxidant enzymes such as dismutase, catalase, and glutathione peroxidase (Tiedge et al., 1997). Thus iron deposition in $\beta$ cells can lead to apoptosis and consequently to decreased insulin synthesis and secretion (Tiedge et al., 1997; Ferrannini, 2000; Cooksey et al., 2004). A recent study in a mouse model of iron overload showed that iron deposition enhances fatty acid oxidation and decreases glucose oxidation in skeletal muscle by inhibiting pyruvate dehydrogenase (PDH) enzyme activity thus increasing IR (Huang etal., 2011). Glucose oxidation is decreased in adipose tissue (Merkel etal., 1988; Green et al., 2006). Iron accumulation also results in an abnormal increase in hepatic glucose production (Mendler et al., 1999; Ferrannini, 2000; Green etal., 2006), inappropriate hepatic insulin extraction, and affects insulin secretion in the pancreas (Niederau et al., 1984). A recent study of 492 subjects demonstrated an association between markers of iron metabolism, adipocyte insulin resistance, and adiponectin (an insulin-sensitizing adipokine), consistent with a model in which iron contributes to T2DM by inducing insulin resistance in adipocytes (Wlazlo et al., 2013). Consistent with this model, mice fed a high iron diet exhibited an accumulation of iron within adipocytes and altered transcription of adipokines involved in glycemic control (Gabrielsen et al., 2012; Dongiovanni etal., 2013). In particular, iron downregulated adiponectin (an adipokine with insulin sensitizing action; Gabrielsen etal., 2012) and increased resistin (an adipokine with hyperglycemic action; Dongiovanni et al., 2013).

The association of dietary iron and diabetes has also been examined. Iron in the diet exists as heme (organic) and nonheme (inorganic) forms. Some studies have shown that the risk of diabetes can be increased by heme iron in the diet (Jiang et al., 2004; Lee et al., 2004; Rajpathak et al., 2006)· Most of these studies were conducted in a healthy US population. In Asian populations, a similar association was reported in a cross sectional study of 2997 Chinese people (Luan de et al., 2008). Consistent results were obtained in an observational cohort of Mediterranean people (Fernandez-Cao et al., 2013). Two recent meta-analyses concluded that higher heme iron poses higher risk of type 2 diabetes (Bao et al., 2012; Zhao et al., 2012). One of the studies reported that there was no significant association with total iron, non-heme iron or iron supplements in the diet (Bao etal., 2012). However, these studies did not separate heme iron per se from other components of red meat. Red meat bears a high correlation with heme iron and has been shown to be associated with the risk of 


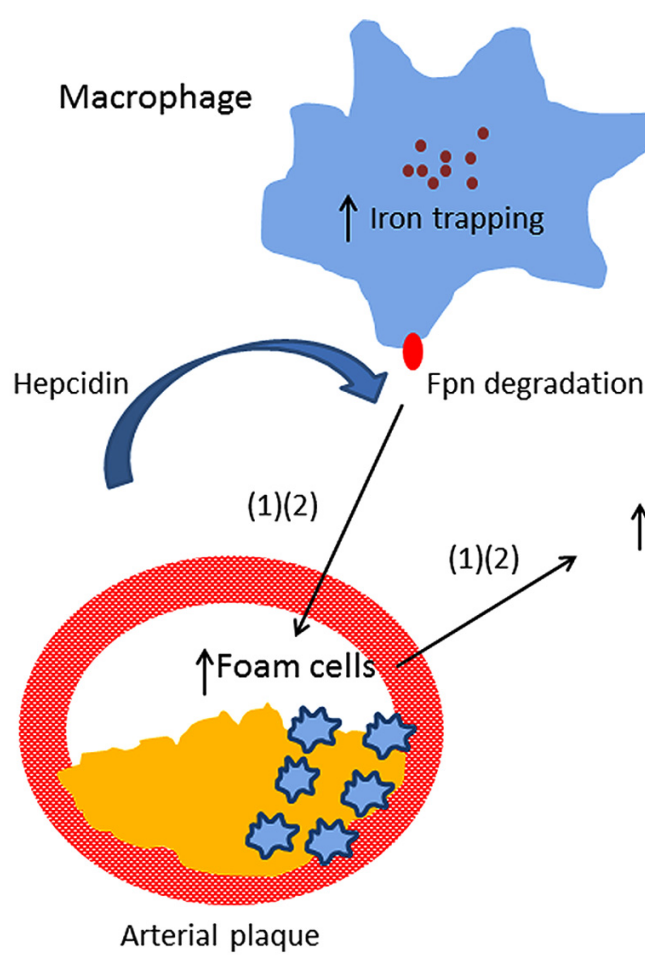

\section{Peroxidation of lipids \\ ROS \\ Inflammation \\ $\downarrow$ \\ Instability of plaque \\ $\downarrow$}

Ischemic Events

FIGURE 1 | Model showing iron retention in macrophages promotes arterial plaque destabilization (Sullivan, 2007; Theurl et al., 2008).

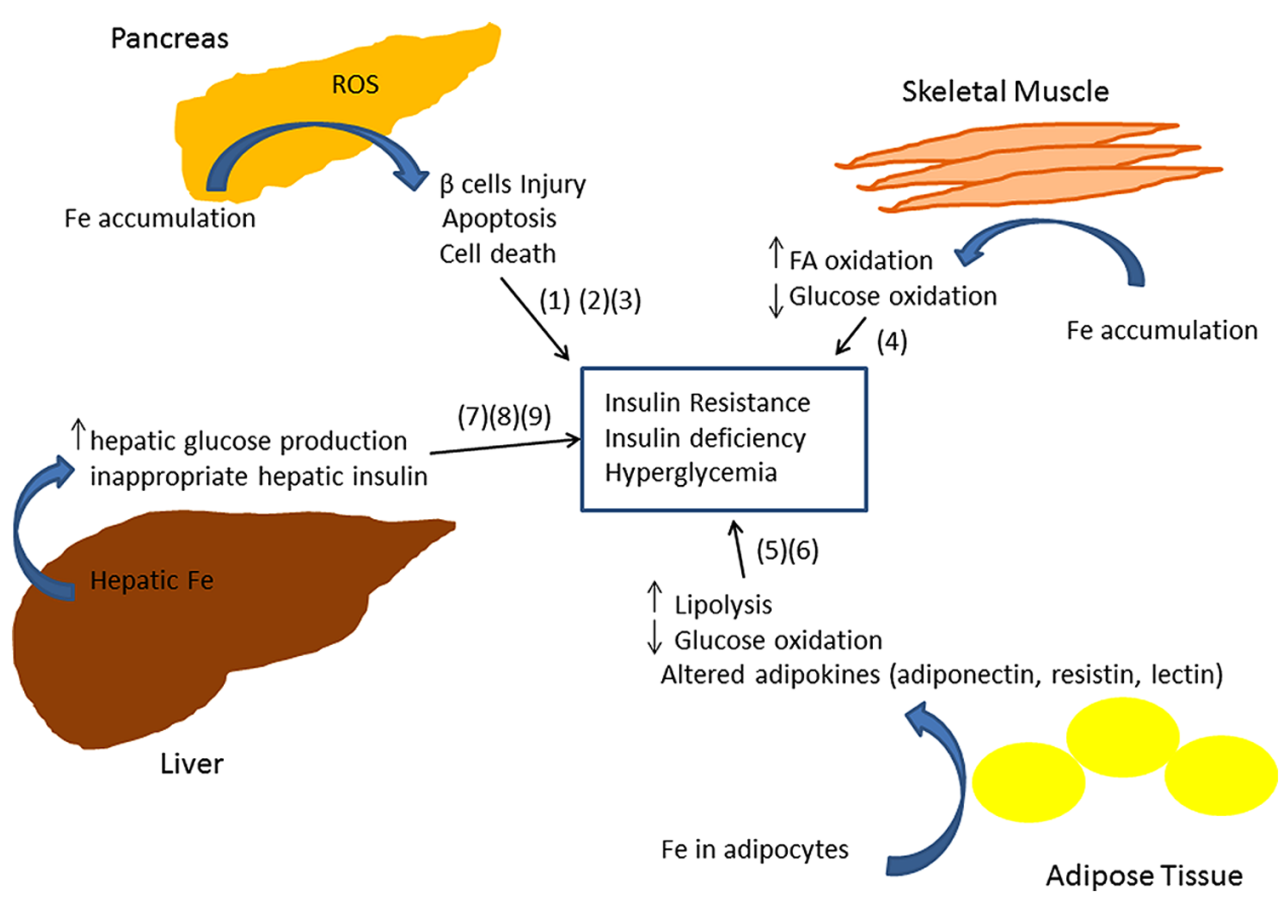

FIGURE 2 | Model showing multiple mechanisms through which iron can lead to insulin resistance and insufficiency (Merkel et al., 1988; Tiedge et al., 1997; Mendler et al., 1999; Ferrannini, 2000; Cooksey et al., 2004; Green et al., 2006; Huang et al., 2011). 
type 2 diabetes (Pan et al., 2011). Therefore confounding by other components from red meat cannot be ruled out with certainty. Screening for HFE mutation was not conducted in these studies, and hence a contribution of genetic interaction cannot be ruled out either.

Despite the limitations of epidemiological studies, intervention studies support the association between excess iron, metabolic syndrome and diabetes. Iron reduction by phlebotomy and chelation therapy produced an improvement in glucose tolerance not only in patients with hemochromatosis (Dymock et al., 1972; Inoue et al., 1997), but also in healthy donors. Houschyar et al. (2012) reported that reduction of body iron stores through phlebotomy had therapeutic effects in metabolic syndrome patients, including lowered blood pressure and improvement in glycemic control and cardiovascular risks. Fernandez-Real et al. (2002) found that bloodletting in high ferritin type 2 diabetes patients resulted in decrease in glycated hemoglobin and also improved insulin secretion and sensitivity.

\section{CONCLUSION}

Epidemiological studies provide evidence that elevated iron stores are a risk factor for developing cardiovascular and metabolic abnormalities. Such results have been verified in diverse ethnic and geographic populations. Although mechanistic insights have been limited, iron-dependent pathophysiological pathways involved in these two conditions may exhibit some differences. In diabetes and metabolic syndrome, iron may contribute to risk following deposition in the liver, pancreas, and skeletal muscle, where it can enhance oxidative damage and contribute to insulin deficiency and resistance. In CVD, iron within macrophages and foam cells predisposes to the formation of atherosclerotic plaques. Hepcidin may promote plaque destabilization by preventing iron export from the intralesional macrophages leading to ischemic events. Although additional mechanisms are likely involved, Figures 1 and 2 illustrate some pathways through which excess iron can increase risk of CVD, metabolic syndrome, and diabetes.

Regarding iron in the diet, there is still insufficient data to formulate guidelines on dietary iron restrictions in the at-risk or general population. This is primarily because dietary iron exists in two very different forms - heme and non-heme iron. Study findings are more inclined toward the association of heme iron (mainly from meat) and disease risk rather than non-heme iron. Although some studies have attempted to assess whether iron supplementation is linked to disease risk, particularly diabetes in women, the results have been inconsistent (Rajpathak et al., 2006; Bo et al., 2009; Chan et al., 2009).

Further research is required to identify more predictors of body iron stores that may help in reducing the risk of cardiovascular or metabolic disease. Experiments are needed to unravel the underlying biological mechanism of this association. Additionally, more randomized controlled studies are warranted to evaluate the clinical outcome of patients placed on iron restricted diets or subjected to iron depletion therapy so that therapeutic recommendations can be made.

\section{ACKNOWLEDGMENT}

Supported in part by NIH R01 CA171101 (Frank M. Torti).

\section{REFERENCES}

Andrews, N. C. (1999). Disorders of iron metabolism. N. Engl. J. Med. 341, 19861995. doi: 10.1056/NEJM199912233412607

Angulo, P. (2002). Nonalcoholic fatty liver disease. N. Engl. J. Med. 346, 1221-1231. doi: 10.1056/NEJMra011775

Baer, D. M., Tekawa, I. S., and Hurley, L. B. (1994). Iron stores are not associated with acute myocardial infarction. Circulation 89, 2915-2918. doi: 10.1161/01.CIR.89.6.2915

Bao, W., Rong, Y., Rong, S., and Liu, L. (2012). Dietary iron intake, body iron stores, and the risk of type 2 diabetes: a systematic review and meta-analysis. $B M C \mathrm{Med}$. 10:119. doi: 10.1186/1741-7015-10-119

Barisani, D., Pelucchi, S., Mariani, R., Galimberti, S., Trombini, P., Fumagalli, D., et al. (2008). Hepcidin and iron-related gene expression in subjects with dysmetabolic hepatic iron overload. J. Hepatol. 49, 123-133. doi: 10.1016/j.jhep.2008.03.011

Bekri, S., Gual, P., Anty, R., Luciani, N., Dahman, M., Ramesh, B., et al. (2006). Increased adipose tissue expression of hepcidin in severe obesity is independent from diabetes and NASH. Gastroenterology 131, 788-796. doi: 10.1053/j.gastro.2006.07.007

Benson, K., and Hartz, A. J. (2000). A comparison of observational studies and randomized, controlled trials. N. Engl. J. Med. 342, 1878-1886. doi: 10.1056/NEJM200006223422506

Berliner, J. A., and Heinecke, J. W. (1996). The role of oxidized lipoproteins in atherogenesis. Free Radic. Biol. Med. 20, 707-727. doi: 10.1016/0891-5849(95) 02173-6

Bo, S., Menato, G., Villois, P., Gambino, R., Cassader, M., Cotrino, I., et al. (2009). Iron supplementation and gestational diabetes in midpregnancy. Am. J. Obstet. Gynecol. 201, 158.e1-158.e6. doi: 10.1016/j.ajog.2009.04.049

Bottiger, L. E., and Carlson, L. A. (1982). Risk factors for death for males and females. A study of the death pattern in the Stockholm prospective study. Acta Med. Scand. 211, 437-442. doi: 10.1111/j.0954-6820.1982.tb01978.x

Bozzini, C., Girelli, D., Olivieri, O., Martinelli, N., Bassi, A., De Matteis, G., et al. (2005). Prevalence of body iron excess in the metabolic syndrome. Diabetes Care 28, 2061-2063. doi: 10.2337/diacare.28.8.2061

Castellanos, M., Puig, N., Carbonell, T., Castillo, J., Martinez, J., Rama, R., et al. (2002). Iron intake increases infarct volume after permanent middle cerebral artery occlusion in rats. Brain Res. 952, 1-6. doi: 10.1016/S0006-8993(02) 03179-7

Chan, K. K., Chan, B. C., Lam, K. F., Tam, S., and Lao, T. T. (2009). Iron supplement in pregnancy and development of gestational diabetes - a randomised placebocontrolled trial. BJOG 116, 789-797; discussion 797-788. doi: 10.1111/j.14710528.2008.02014.x

Choi, K. M., Lee, K. W., Kim, H. Y., Seo, J. A., Kim, S. G., Kim, N. H., et al. (2005). Association among serum ferritin, alanine aminotransferase levels, and metabolic syndrome in Korean postmenopausal women. Metabolism 54, 1510-1514. doi: 10.1016/j.metabol.2005.05.018

Concato, J., Shah, N., and Horwitz, R. I. (2000). Randomized, controlled trials, observational studies, and the hierarchy of research designs. N. Engl. J. Med. 342, 1887-1892. doi: 10.1056/NEJM200006223422507

Cook, J. D., Lipschitz, D. A., Miles, L. E., and Finch, C. A. (1974). Serum ferritin as a measure of iron stores in normal subjects. Am. J. Clin. Nutr. 27, 681-687.

Cooksey, R. C., Jouihan, H. A., Ajioka, R. S., Hazel, M. W., Jones, D. L., Kushner, J. P., et al. (2004). Oxidative stress, beta-cell apoptosis, and decreased insulin secretory capacity in mouse models of hemochromatosis. Endocrinology 145, 5305-5312. doi: 10.1210/en.2004-0392

Cullen, K. J., Stenhouse, N. S., and Wearne, K. L. (1981). Raised haemoglobin and risk of cardiovascular disease. Lancet 2, 1288-1289. doi: 10.1016/S01406736(81)91524-5

Danesh, J., and Appleby, P. (1999). Coronary heart disease and iron status: meta-analyses of prospective studies. Circulation 99, 852-854. doi: 10.1161/01.CIR.99.7.852

Datz, C., Felder, T. K., Niederseer, D., and Aigner, E. (2013). Iron homeostasis in the metabolic syndrome. Eur. J. Clin. Invest. 43, 215-224. doi: 10.1111/eci. 12032

Dongiovanni, P., Fracanzani, A. L., Fargion, S., and Valenti, L. (2011). Iron in fatty liver and in the metabolic syndrome: a promising therapeutic target. J. Hepatol. 55, 920-932. doi: 10.1016/j.jhep.2011.05.008 
Dongiovanni, P., Ruscica, M., Rametta, R., Recalcati, S., Steffani, L., Gatti, S., et al. (2013). Dietary iron overload induces visceral adipose tissue insulin resistance. Am. J. Pathol. 182, 2254-2263. doi: 10.1016/j.ajpath.2013.02.019

Dunn, T., Blankenship, D., Beal, N., Allen, R., Schechter, E., Moore, W., et al. (2008). HFE mutations in heart disease. Heart Vessels 23, 348-355. doi: 10.1007/s00380008-1047-8

Dymock, I. W., Cassar, J., Pyke, D. A., Oakley, W. G., and Williams, R. (1972). Observations on the pathogenesis, complications and treatment of diabetes in 115 cases of haemochromatosis. Am. J. Med. 52, 203-210. doi: 10.1016/00029343(72)90070-8

Engberink, M. F., Povel, C. M., Durga, J., Swinkels, D. W., De Kort, W. L., Schouten, E. G., et al. (2010). Hemochromatosis (HFE) genotype and atherosclerosis: increased susceptibility to iron-induced vascular damage in C282Y carriers? Atherosclerosis 211, 520-525. doi: 10.1016/j.atherosclerosis. 2010 03.018

Fernandez-Cao, J. C., Arija, V., Aranda, N., Bullo, M., Basora, J., MartinezGonzalez, M. A., et al. (2013). Heme iron intake and risk of new-onset diabetes in a Mediterranean population at high risk of cardiovascular disease: an observational cohort analysis. BMC Public Health 13:1042. doi: 10.1186/1471-245813-1042

Fernandez-Real, J. M., Penarroja, G., Castro, A., Garcia-Bragado, F., HernandezAguado, I., and Ricart, W. (2002). Blood letting in high-ferritin type 2 diabetes: effects on insulin sensitivity and beta-cell function. Diabetes 51, 1000-1004. doi: 10.2337/diabetes.51.4.1000

Ferrannini, E. (2000). Insulin resistance, iron, and the liver. Lancet 355, 2181-2182. doi: 10.1016/S0140-6736(00)02397-7

Forouhi, N. G., Harding, A. H., Allison, M., Sandhu, M. S., Welch, A., Luben, R., et al. (2007). Elevated serum ferritin levels predict new-onset type 2 diabetes: results from the EPIC-Norfolk prospective study. Diabetologia 50, 949-956. doi: 10.1007/s00125-007-0604-5

Forstermann, U. (2008). Oxidative stress in vascular disease: causes, defense mechanisms and potential therapies. Nat. Clin. Pract. Cardiovasc. Med. 5, 338-349. doi: 10.1038/ncpcardio1211

Friedrich, N., Milman, N., Volzke, H., Linneberg, A., and Jorgensen, T. (2009). Is serum ferritin within the reference range a risk predictor of cardiovascular disease? A population-based, long-term study comprising 2874 subjects. Br. J. Nutr. 102, 594-600. doi: 10.1017/S000711450922085X

Gabrielsen, J. S., Gao, Y., Simcox, J. A., Huang, J., Thorup, D., Jones, D., et al. (2012) Adipocyte iron regulates adiponectin and insulin sensitivity. J. Clin. Invest. 122, 3529-3540. doi: 10.1172/JCI44421

Gaenzer, H., Marschang, P., Sturm, W., Neumayr, G., Vogel, W., Patsch, J., et al. (2002). Association between increased iron stores and impaired endothelial function in patients with hereditary hemochromatosis. J. Am. Coll. Cardiol. 40, 2189-2194. doi: 10.1016/S0735-1097(02)02611-6

Ganz, T. (2013). Systemic iron homeostasis. Physiol. Rev. 93, 1721-1741. doi 10.1152/physrev.00008.2013

Go, A. S., Mozaffarian, D., Roger, V. L., Benjamin, E. J., Berry, J. D., Borden, W. B., et al. (2013). Heart disease and stroke statistics-2013 update: a report from the American Heart Association. Circulation 127, e6-e245. doi: 10.1161/CIR.0b013e318282ab8f

Gonzalez, A. S., Guerrero, D. B., Soto, M. B., Diaz, S. P., Martinez-Olmos, M., and Vidal, O. (2006). Metabolic syndrome, insulin resistance and the inflammation markers C-reactive protein and ferritin. Eur. J. Clin. Nutr. 60, 802-809. doi 10.1038/sj.ejcn.1602384

Gordon, T., Kannel, W. B., Hjortland, M. C., and Mcnamara, P. M. (1978) Menopause and coronary heart disease. The Framingham Study. Ann. Intern. Med. 89, 157-161. doi: 10.7326/0003-4819-89-2-157

Green, A., Basile, R., and Rumberger, J. M. (2006). Transferrin and iron induce insulin resistance of glucose transport in adipocytes. Metabolism 55, 1042-1045. doi: 10.1016/j.metabol.2006.03.015

Greenberg, A. S., and Obin, M. S. (2006). Obesity and the role of adipose tissue in inflammation and metabolism. Am. J. Clin. Nutr. 83, 461S-465S.

Gupta, R., Rastogi, S., Nagar, R., Kastia, S., and Kaul, V. (2000). Dietary and serum iron, body iron stores and coronary heart disease. J. Assoc. Physicians India 48 489-492.

Halle, M., Konig, D., Berg, A., Keul, J., and Baumstark, M. W. (1997). Relationship of serum ferritin concentrations with metabolic cardiovascular risk factors in men without evidence for coronary artery disease. Atherosclerosis 128, 235-240. doi: 10.1016/S0021-9150(96)05994-1

Heidenreich, P. A., Trogdon, J. G., Khavjou, O. A., Butler, J., Dracup, K., Ezekowitz, M. D., et al. (2011). Forecasting the future of cardiovascular disease in the United States: a policy statement from the American Heart Association. Circulation 123, 933-944. doi: 10.1161/CIR.0b013e31820a55f5

Heron, M., Hoyert, D. L., Murphy, S. L., Xu, J., Kochanek, K. D., and Tejada-Vera, B. (2009). Deaths: final data for 2006. Natl. Vital. Stat. Rep. 57, 1-134.

Houschyar, K. S., Ludtke, R., Dobos, G. J., Kalus, U., Broecker-Preuss, M., Rampp, T., et al. (2012). Effects of phlebotomy-induced reduction of body iron stores on metabolic syndrome: results from a randomized clinical trial. BMC Med. 10:54. doi: 10.1186/1741-7015-10-54

Huang, J., Jones, D., Luo, B., Sanderson, M., Soto, J., Abel, E. D., et al. (2011). Iron overload and diabetes risk: a shift from glucose to Fatty Acid oxidation and increased hepatic glucose production in a mouse model of hereditary hemochromatosis. Diabetes Metab. Res. Rev. 60, 80-87. doi: 10.2337/db 10-0593

Inoue, Y., Nakanishi, K., Hiraga, T., Okubo, M., Murase, T., Kosaka, K., et al. (1997). Recovery of pancreatic beta-cell function in hemochromatosis: combined treatment with recombinant human erythropoietin and phlebotomy. Am. J. Med. Sci. 314, 401-402. doi: 10.1097/00000441-199712000-00008

Jacobs, A., Miller, F., Worwood, M., Beamish, M. R., and Wardrop, C. A. (1972). Ferritin in the serum of normal subjects and patients with iron deficiency and iron overload. Br. Med. J. 4, 206-208. doi: 10.1136/bmj.4. 5834.206

Jacobs, A., and Worwood, M. (1975). Ferritin in serum. Clinical and biochemical implications. N. Engl. J. Med. 292, 951-956. doi: 10.1056/NEJM1975050129 21805

Jehn, M., Clark, J. M., and Guallar, E. (2004). Serum ferritin and risk of the metabolic syndrome in U.S. adults. Diabetes Care 27, 2422-2428. doi: $10.2337 /$ diacare.27.10.2422

Jehn, M. L., Guallar, E., Clark, J. M., Couper, D., Duncan, B. B., Ballantyne, C. M., et al. (2007). A prospective study of plasma ferritin level and incident diabetes: the Atherosclerosis Risk in Communities (ARIC) Study. Am. J. Epidemiol. 165, 1047-1054. doi: 10.1093/aje/kwk093

Jiang, R., Manson, J. E., Meigs, J. B., Ma, J., Rifai, N., and Hu, F. B. (2004). Body iron stores in relation to risk of type 2 diabetes in apparently healthy women. JAMA 291, 711-717. doi: 10.1001/jama.291.6.711

Kaltwasser, J. P., and Werner, E. (1989). Diagnosis and clinical evaluation of iron overload. Baillieres Clin. Haematol. 2, 363-389. doi: 10.1016/S09503536(89)80022-8

Kaluza, J., Wolk, A., and Larsson, S. C. (2013). Heme iron intake and risk of stroke: a prospective study of men. Stroke 44, 334-339. doi: 10.1161/STROKEAHA.112.679662

Kautz, L., Gabayan, V., Wang, X., Wu, J., Onwuzurike, J., Jung, G., et al. (2013). Testing the iron hypothesis in a mouse model of atherosclerosis. Cell Rep. 5, 1436-1442. doi: 10.1016/j.celrep.2013.11.009

Kiechl, S., Willeit, J., Egger, G., Poewe, W., and Oberhollenzer, F. (1997). Body iron stores and the risk of carotid atherosclerosis: prospective results from the Bruneck study. Circulation 96, 3300-3307. doi: 10.1161/01.CIR.96.10.3300

Knottnerus, J. A., Swaen, G. M., Slangen, J. J., Volovics, A., and Durinck, J. (1988). Haematologic parameters as risk factors for cardiac infarction, in an occupational health care setting. J. Clin. Epidemiol. 41, 67-74. doi: 10.1016/0895-4356(88)90010-8

Knuiman, M. W., Divitini, M. L., Olynyk, J. K., Cullen, D. J., and Bartholomew, H. C. (2003). Serum ferritin and cardiovascular disease: a 17-year follow-up study in Busselton, Western Australia. Am. J. Epidemiol. 158, 144-149. doi: 10.1093/aje/kwg121

Lee, C. C., Adler, A. I., Sandhu, M. S., Sharp, S. J., Forouhi, N. G., Erqou, S., et al. (2009). Association of C-reactive protein with type 2 diabetes: prospective analysis and meta-analysis. Diabetologia 52, 1040-1047. doi: 10.1007/s00125-009-1338-3

Lee, D. H., Folsom, A. R., and Jacobs, D. R. Jr. (2004). Dietary iron intake and Type 2 diabetes incidence in postmenopausal women: the Iowa Women's Health Study. Diabetologia 47, 185-194. doi: 10.1007/s00125-003-1307-1

Li, J., Wang, R., Luo, D., Li, S., and Xiao, C. (2013). Association between serum ferritin levels and risk of the metabolic syndrome in Chinese adults: a population study. PLoS ONE 8:e74168. doi: 10.1371/journal.pone.0074168 
Luan de, C., Li, H., Li, S. J., Zhao, Z., Li, X., and Liu, Z. M. (2008). Body iron stores and dietary iron intake in relation to diabetes in adults in North China. Diabetes Care 31, 285-286. doi: 10.2337/dc07-0921

Marchesini, G., Brizi, M., Bianchi, G., Tomassetti, S., Bugianesi, E., Lenzi, M., et al. (2001). Nonalcoholic fatty liver disease: a feature of the metabolic syndrome. Diabetes Metab. Res. Rev. 50, 1844-1850.

Marchesini, G., Bugianesi, E., Forlani, G., Cerrelli, F., Lenzi, M., Manini, R., et al. (2003). Nonalcoholic fatty liver, steatohepatitis, and the metabolic syndrome. Hepatology 37, 917-923. doi: 10.1053/jhep.2003.50161

McCord, J. M. (1991). Is iron sufficiency a risk factor in ischemic heart disease? Circulation 83, 1112-1114. doi: 10.1161/01.CIR.83.3.1112

Mendler, M. H., Turlin, B., Moirand, R., Jouanolle, A. M., Sapey, T., Guyader, D., et al. (1999). Insulin resistance-associated hepatic iron overload. Gastroenterology 117, 1155-1163. doi: 10.1016/S0016-5085(99)70401-4

Merkel, P. A., Simonson, D. C., Amiel, S. A., Plewe, G., Sherwin, R. S., Pearson, H. A., et al. (1988). Insulin resistance and hyperinsulinemia in patients with thalassemia major treated by hypertransfusion. N. Engl. J. Med. 318, 809-814. doi: 10.1056/NEJM198803313181303

Montonen, J., Boeing, H., Steffen, A., Lehmann, R., Fritsche, A., Joost, H. G. et al. (2012). Body iron stores and risk of type 2 diabetes: results from the European Prospective Investigation into Cancer and Nutrition (EPIC)-Potsdam study. Diabetologia 55, 2613-2621. doi: 10.1007/s00125-012-2633-y

Nelson, C. W., Wei, E. P., Povlishock, J. T., Kontos, H. A., and Moskowitz, M. A. (1992). Oxygen radicals in cerebral ischemia. Am. J. Physiol. 263, H1356-H1362.

Nemeth, E., Tuttle, M. S., Powelson, J., Vaughn, M. B., Donovan, A., Ward D. M., et al. (2004). Hepcidin regulates cellular iron efflux by binding to ferroportin and inducing its internalization. Science 306, 2090-2093. doi 10.1126/science. 1104742

Niederau, C., Berger, M., Stremmel, W., Starke, A., Strohmeyer, G., Ebert, R., et al. (1984). Hyperinsulinaemia in non-cirrhotic haemochromatosis: impaired hepatic insulin degradation? Diabetologia 26, 441-444. doi: 10.1007/BF002 62217

Pan, A., Sun, Q., Bernstein, A. M., Schulze, M. B., Manson, J. E., Willett, W. C., et al. (2011). Red meat consumption and risk of type 2 diabetes: 3 cohorts of US adults and an updated meta-analysis. Am. J. Clin. Nutr. 94, 1088-1096. doi: 10.3945/ajen.111.018978

Paraskevaidis, I. A., Iliodromitis, E. K., Vlahakos, D., Tsiapras, D. P., Nikolaidis A., Marathias, A., et al. (2005). Deferoxamine infusion during coronary artery bypass grafting ameliorates lipid peroxidation and protects the myocardium against reperfusion injury: immediate and long-term significance. Eur. Heart J. 26, 263-270. doi: 10.1093/eurheartj/ehi028

Powell, L. W., George, D. K., McDonnell, S. M., and Kowdley, K. V. (1998). Diagnosis of hemochromatosis. Ann. Intern. Med. 129, 925-931. doi: 10.7326/0003-4819129-11_Part_2-199812011-00002

Rajapurkar, M. M., Shah, S. V., Lele, S. S., Hegde, U. N., Lensing, S. Y., Gohel, K., et al. (2012). Association of catalytic iron with cardiovascular disease. Am. J. Cardiol. 109, 438-442. doi: 10.1016/j.amjcard.2011.09.032

Rajpathak, S., Ma, J., Manson, J., Willett, W. C., and Hu, F. B. (2006). Iron intake and the risk of type 2 diabetes in women: a prospective cohort study. Diabetes Care 29, 1370-1376. doi: 10.2337/dc06-0119

Rajpathak, S. N., Crandall, J. P., Wylie-Rosett, J., Kabat, G. C., Rohan, T. E., and Hu, F. B. (2009). The role of iron in type 2 diabetes in humans. Biochim. Biophys. Acta 1790, 671-681. doi: 10.1016/j.bbagen.2008.04.005

Riva, A., Trombini, P., Mariani, R., Salvioni, A., Coletti, S., Bonfadini, S., et al. (2008). Revaluation of clinical and histological criteria for diagnosis of dysmetabolic iron overload syndrome. World J. Gastroenterol. 14, 4745-4752. doi: 10.3748/wjg. 14.4745

Roest, M., Van Der Schouw, Y. T., De Valk, B., Marx, J. J., Tempelman, M. J., De Groot, P. G., et al. (1999). Heterozygosity for a hereditary hemochromatosis gene is associated with cardiovascular death in women. Circulation 100, 1268-1273. doi: 10.1161/01.CIR.100.12.1268

Salonen, J. T., Nyyssonen, K., Korpela, H., Tuomilehto, J., Seppanen, R., and Salonen, R. (1992a). High stored iron levels are associated with excess risk of myocardial infarction in eastern Finnish men. Circulation 86, 803-811. doi: 10.1161/01.CIR.86.3.803

Salonen, J. T., Yla-Herttuala, S., Yamamoto, R., Butler, S., Korpela, H., Salonen, R., et al. (1992b). Autoantibody against oxidised LDL and progression of carotid atherosclerosis. Lancet 339, 883-887. doi: 10.1016/0140-6736(92)90926-T
Salonen, J. T., Tuomainen, T. P., Nyyssonen, K., Lakka, H. M., and Punnonen, K. (1998). Relation between iron stores and non-insulin dependent diabetes in men: case-control study. BMJ 317:727. doi: 10.1136/bmj.317. 7160.727

Skikne, B. S., Flowers, C. H., and Cook, J. D. (1990). Serum transferrin receptor: a quantitative measure of tissue iron deficiency. Blood 75, 1870-1876.

Song, J. W., and Chung, K. C. (2010). Observational studies: cohort and case-control studies. Plast. Reconstr. Surg. 126, 2234-2242. doi: 10.1097/PRS.0b013e3181f44abc

Steen, D. L., Cannon, C. P., Lele, S. S., Rajapurkar, M. M., Mukhopadhyay, B., Scirica, B. M., et al. (2013). Prognostic evaluation of catalytic iron in patients with acute coronary syndromes. Clin. Cardiol. 36, 139-145. doi: 10.1002/clc. 22089

Sullivan, J. L. (1981). Iron and the sex difference in heart disease risk. Lancet 1, 1293-1294. doi: 10.1016/S0140-6736(81)92463-6

Sullivan, J. L. (1989). The iron paradigm of ischemic heart disease. Am. Heart J. 117, 1177-1188. doi: 10.1016/0002-8703(89)90887-9

Sullivan, J. L. (2007). Macrophage iron, hepcidin, and atherosclerotic plaque stability. Exp. Biol. Med. (Maywood) 232, 1014-1020. doi: 10.3181/0703MR-54

Sullivan, J. L. (2009). Iron in arterial plaque: modifiable risk factor for atherosclerosis. Biochim. Biophys. Acta 1790, 718-723. doi: 10.1016/j.bbagen.2008. 06.005

Sun, L., Franco, O. H., Hu, F. B., Cai, L., Yu, Z., Li, H., et al. (2008a). Ferritin concentrations, metabolic syndrome, and type 2 diabetes in middle-aged and elderly chinese. J. Clin. Endocrinol. Metab. 93, 4690-4696. doi: 10.1210/jc.20081159

Sun, Q., Ma, J., Rifai, N., Franco, O. H., Rexrode, K. M., and Hu, F. B. (2008b). Excessive body iron stores are not associated with risk of coronary heart disease in women. J. Nutr. 138, 2436-2441. doi: 10.3945/jn.108. 097766

Sun, L., Zong, G., Pan, A., Ye, X., Li, H., Yu, Z., et al. (2013). Elevated plasma ferritin is associated with increased incidence of type 2 diabetes in middleaged and elderly Chinese adults. J. Nutr. 143, 1459-1465. doi: 10.3945/jn.113. 177808

Sung, K. C., Kang, S. M., Cho, E. J., Park, J. B., Wild, S. H., and Byrne, C. D. (2012). Ferritin is independently associated with the presence of coronary artery calcium in 12,033 men. Arterioscler. Thromb. Vasc. Biol. 32, 2525-2530. doi: 10.1161/ATVBAHA.112.253088

Theurl, I., Theurl, M., Seifert, M., Mair, S., Nairz, M., Rumpold, H., et al. (2008). Autocrine formation of hepcidin induces iron retention in human monocytes. Blood 111, 2392-2399. doi: 10.1182/blood-2007-05-090019

Thomas, C. E., Morehouse, L. A., and Aust, S. D. (1985). Ferritin and superoxidedependent lipid peroxidation. J. Biol. Chem. 260, 3275-3280.

Tiedge, M., Lortz, S., Drinkgern, J., and Lenzen, S. (1997). Relation between antioxidant enzyme gene expression and antioxidative defense status of insulin-producing cells. Diabetes 46, 1733-1742. doi: 10.2337/diab.46. 11.1733

Tuomainen, T. P., Punnonen, K., Nyyssonen, K., and Salonen, J. T. (1998). Association between body iron stores and the risk of acute myocardial infarction in men. Circulation 97, 1461-1466. doi: 10.1161/01.CIR.97.15.1461

Turoczi, T., Jun, L., Cordis, G., Morris, J. E., Maulik, N., Stevens, R. G., et al. (2003). HFE mutation and dietary iron content interact to increase ischemia/reperfusion injury of the heart in mice. Circ. Res. 92, 1240-1246. doi: 10.1161/01.RES.0000076890.59807.23

Valenti, L., Dongiovanni, P., and Fargion, S. (2012). Diagnostic and therapeutic implications of the association between ferritin level and severity of nonalcoholic fatty liver disease. World J. Gastroenterol. 18, 3782-3786. doi: 10.3748/wjg.v18.i29.3782

Valenti, L., Dongiovanni, P., Fracanzani, A. L., Santorelli, G., Fatta, E., Bertelli, C., etal. (2003). Increased susceptibility to nonalcoholic fatty liver disease in heterozygotes for the mutation responsible for hereditary hemochromatosis. Dig. Liver Dis. 35, 172-178. doi: 10.1016/S1590-8658(03) 00025-2

Valenti, L., Dongiovanni, P., Piperno, A., Fracanzani, A. L., Maggioni, M., Rametta, R., et al. (2006). Alpha 1-antitrypsin mutations in NAFLD: high prevalence and association with altered iron metabolism but not with liver damage. Hepatology 44, 857-864. doi: 10.1002/hep. 21329 
Valenti, L., Fracanzani, A. L., Bugianesi, E., Dongiovanni, P., Galmozzi, E., Vanni, E., et al. (2010). HFE genotype, parenchymal iron accumulation, and liver fibrosis in patients with nonalcoholic fatty liver disease. Gastroenterology 138, 905-912. doi: 10.1053/j.gastro.2009.11.013

Valenti, L., Fracanzani, A. L., Dongiovanni, P., Bugianesi, E., Marchesini, G. Manzini, P., et al. (2007). Iron depletion by phlebotomy improves insulin resistance in patients with nonalcoholic fatty liver disease and hyperferritinemia: evidence from a case-control study. Am. J. Gastroenterol. 102, 1251-1258. doi: 10.1111/j.1572-0241.2007.01192.x

Valenti, L., Fracanzani, A. L., Dongiovanni, P., Rovida, S., Rametta, R., Fatta, E., et al. (2014). A randomized trial of iron depletion in patients with nonalcoholic fatty liver disease and hyperferritinemia. World J. Gastroenterol. 20, 3002-3010. doi 10.3748/wjg.v20.i11.3002

Valenti, L., Swinkels, D. W., Burdick, L., Dongiovanni, P., Tjalsma, H., Motta, B. M., et al. (2011). Serum ferritin levels are associated with vascular damage in patient with nonalcoholic fatty liver disease. Nutr. Metab. Cardiovasc. Dis. 21, 568-575. doi: 10.1016/j.numecd.2010.01.003

van der, A. D., Grobbee, D. E., Roest, M., Marx, J. J., Voorbij, H. A., and Van Der Schouw, Y. T. (2005). Serum ferritin is a risk factor for stroke in postmenopausal women. Stroke 36, 1637-1641. doi: 10.1161/01.STR.0000173172. 82880.72

van der, A. D., Peeters, P. H., Grobbee, D. E., Roest, M., Marx, J. J., Voorbij, H. M., et al. (2006). HFE mutations and risk of coronary heart disease in middle-aged women. Eur. J. Clin. Invest. 36, 682-690. doi: 10.1111/j.1365-2362.2006.01711.x

von Bonsdorff, L., Lindeberg, E., Sahlstedt, L., Lehto, J., and Parkkinen, J. (2002) Bleomycin-detectable iron assay for non-transferrin-bound iron in hematologic malignancies. Clin. Chem. 48, 307-314.

Wang, W., Knovich, M. A., Coffman, L. G., Torti, F. M., and Torti, S. V. (2010). Serum ferritin: past, present and future. Biochim. Biophys. Acta 1800, 760-769. doi: 10.1016/j.bbagen.2010.03.011

Williams, R. E., Zweier, J. L., and Flaherty, J. T. (1991). Treatment with deferoxamine during ischemia improves functional and metabolic recovery and reduces reperfusion-induced oxygen radical generation in rabbit hearts. Circulation 83, 1006-1014. doi: 10.1161/01.CIR.83.3.1006

Witte, D. L., Crosby, W. H., Edwards, C. Q., Fairbanks, V. F., and Mitros, F. A. (1996). Practice guideline development task force of the College of American Pathologists. Hereditary hemochromatosis. Clin. Chim. Acta 245, 139-200. doi: 10.1016/0009-8981(95)06212-2

Wlazlo, N., Van Greevenbroek, M. M., Ferreira, I., Jansen, E. H., Feskens, E. J., Van Der Kallen, C. J., et al. (2013). Iron metabolism is associated with adipocyte insulin resistance and plasma adiponectin: the Cohort on Diabetes and Atherosclerosis Maastricht (CODAM) study. Diabetes Care 36, 309-315. doi: $10.2337 / \mathrm{dc} 12-0505$

Wolff, B., Volzke, H., Ludemann, J., Robinson, D., Vogelgesang, D., Staudt, A., et al. (2004). Association between high serum ferritin levels and carotid atherosclerosis in the study of health in Pomerania (SHIP). Stroke 35, 453-457. doi: 10.1161/01.STR.0000114875.31599.1C

Wolff, S. P. (1993). Diabetes mellitus and free radicals. Free radicals, transition metals and oxidative stress in the aetiology of diabetes mellitus and complications. $\mathrm{Br}$. Med. Bull. 49, 642-652.

Wrede, C. E., Buettner, R., Bollheimer, L. C., Scholmerich, J., Palitzsch, K. D., and Hellerbrand, C. (2006). Association between serum ferritin and the insulin resistance syndrome in a representative population. Eur. J. Endocrinol. 154, 333340. doi: 10.1530/eje.1.02083

Zacharski, L. R., Shamayeva, G., and Chow, B. K. (2011). Effect of controlled reduction of body iron stores on clinical outcomes in peripheral arterial disease. Am. Heart J. 162, 949.e1-957.e1. doi: 10.1016/j.ahj.2011.08.013

Zhang, W., Iso, H., Ohira, T., Date, O. C., Tanabe, N., Kikuchi, S., et al. (2012). Associations of dietary iron intake with mortality from cardiovascular disease: the JACC study. J. Epidemiol. 22, 484-493. doi: 10.2188/jea.JE20120006

Zhao, Z., Li, S., Liu, G., Yan, F., Ma, X., Huang, Z., et al. (2012). Body iron stores and heme-iron intake in relation to risk of type 2 diabetes: a systematic review and meta-analysis. PLoS ONE 7:e41641. doi: 10.1371/journal.pone.0041641

Conflict of Interest Statement: The authors declare that the research was conducted in the absence of any commercial or financial relationships that could be construed as a potential conflict of interest.

Received: 10 March 2014; accepted: 30 April 2014; published online: 20 May 2014. Citation: Basuli D, Stevens RG, Torti FM and Torti SV (2014) Epidemiological associations between iron and cardiovascular disease and diabetes. Front. Pharmacol. 5:117. doi: 10.3389/fphar.2014.00117

This article was submitted to Drug Metabolism and Transport, a section of the journal Frontiers in Pharmacology.

Copyright (c) 2014 Basuli, Stevens, Torti and Torti. This is an open-access article distributed under the terms of the Creative Commons Attribution License (CC BY). The use, distribution or reproduction in other forums is permitted, provided the original author(s) or licensor are credited and that the original publication in this journal is cited, in accordance with accepted academic practice. No use, distribution or reproduction is permitted which does not comply with these terms. 